\title{
Optoelectronic applications of LTMBE III-V materials
}

\author{
John F. Whitaker \\ Center for Ultrafast Optical Science, University of Michigan, 2200 Bonisteel Blvd, Rm. 1006, Ann Arbor, \\ MI 48109-2099 (USA)
}

\begin{abstract}
A review of the application of semiconductor layers grown at low substrate temperatures to ultrafast optoelectronics is presented. The films, grown by molecular beam epitaxy primarily around $200{ }^{\circ} \mathrm{C}$ and subsequently annealed, are demonstrated to have high resistivity, high mobility, an ultrashort carrier lifetime, and a high dielectric breakdown. This combination of properties makes the low-temperature-grown materials perfectly suited for use in high-speed optoelectronic devices. A number of issues which influence the application of these materials, such as growth temperature, use of an annealing process, layer thickness, and optical wavelength, are considered. Examples of low-temperature-grown semiconductor optoelectronic devices, including ultra-high-bandwidth photoconductive detectors, high-sensitivity, highbandwidth MSM photodetectors, and optical temporal analyzers are demonstrated. While the discussion concentrates on low-temperature-grown $\mathrm{GaAs}$, the lattice-mismatched ternary compound $\operatorname{In}_{x} \mathrm{Ga}_{1-x} \mathrm{As} / \mathrm{GaAs}$ is also considered in the context of detection of the longer wavelengths used in optical communications.
\end{abstract}

\section{Introduction}

With the development of technology for future optical communications networks, including soliton transmission in fibres, high-speed laser diodes, fibre lasers and erbium-doped fibre amplifiers comes the suggestion that, within a decade, operating speeds for communications systems will exceed $100 \mathrm{Gbit} \mathrm{s}^{-1}$. This, along with the fact that sources of short-duration optical pulses will be used for ranging, medical imaging, the generation of short bursts of ultraviolet and X-ray radiation, the generation and reception of submillimetre-wave radiation and many other applications, will lead to a significant demand for detectors that can reproduce optical waveforms in the electrical domain. Furthermore, since ultrashort electrical waveforms can be used in the characterization of electronic components such as transmission lines, high-speed devices and integrated circuits, the need for ultrafast response photoswitches [1] which convert d.c. electrical signals to very brief impulses will also be strong. Each of these optoelectronic applications can be best served by a semiconducting material which has a high resistivity, high responsivity (mobility), short carrier lifetime and high dielectric breakdown. When prepared correctly, layers of GaAs and other III-V semiconductors grown by molecular beam epitaxy at low substrate temperatures (i.e. LT-GaAs) have been demonstrated to exhibit all of these criteria simultaneously.

The first photoconductive (PC) detectors on GaAs [2] used a substrate doped with chromium and were demonstrated to have carrier lifetimes as short as 100 ps. When excited by sub-picosecond pulsed lasers, transients with rise times of less than one picosecond could be produced from these detectors. Such waveforms, which appeared virtually as step functions on picosecond time-scales (Fig. 1), had spectra with components in the $\mathrm{THz}$ regime, and the signals were applied in the qualitative characterization of materials and electronic components such as transmission lines and devices [3, 4]. However, for almost every modern application, from detectors to sampling gates to testsignal generators, the capability of generating a short electrical pulse is required. For optical applications,

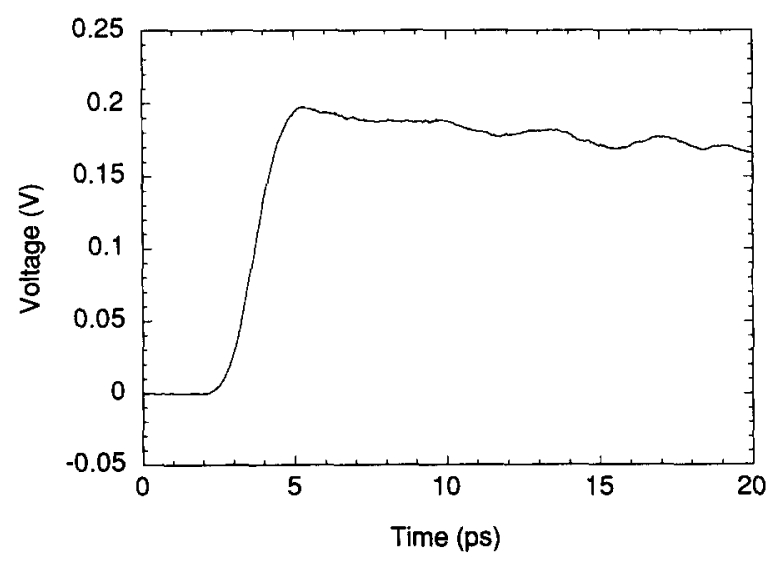

Fig. 1. Photoconductive response of Cr:GaAs. The step-like function is formed due to the long photo-excited carrier lifetime of the semiconductor. 
one wishes to reproduce as faithfully as possible the signal being detected, while for electrical applications, the ability to compute an accurate Fourier transform of a pulsed signal which does not have a long "tail" (which might be corrupted by reflections) is imperative.

A number of approaches have been investigated to achieve short fall times in the response of a detector. These include the identification of photoconductive materials which have very short carrier lifetimes, the fabrication of detector patterns that rely on asymmetric excitation of long carrier lifetime materials [5], and the fabrication of pulse-shaping networks to change the step output of a Cr:GaAs detector into a pulse [6]. Fastlifetime materials utilized have included amorphous silicon [7], films of CdTe [8], $\mathrm{O}^{+}$ion-implanted siliconon-sapphire (SOS) [9] and proton-bombarded GaAs [10] and InP [11]. While the materials that rely on damage and deep-level trapping/recombination centres for their fast response can have very short lifetimes, they also typically have a poor sensitivity to the energy in the excitation pulses, owing to their low mobilities. The materials with higher mobilities do not have the fastest, sub-picosecond relaxation times. The geometrical approaches to producing short pulses have been moderately successful, although they are arguably more difficult to implement. Historically, despite the deficiencies of radiation-damaged SOS, photoconductive detectors fabricated on this material have been used more than any other source to produce picosecond electrical pulses.

\section{Ultrashort carrier lifetime}

In the past several years of its development, LT$\mathrm{GaAs}$ has been found to possess characteristics superior to any other photoconductor. Typically, the optimum GaAs layer for optoelectronics is grown by molecular beam epitaxy at low substrate temperatures $\left(\approx 190-210^{\circ} \mathrm{C}\right)$ at a growth rate of $1 \mu \mathrm{m} \mathrm{h}^{-1}$ [12]. This substrate temperature, the group III-V flux ratio, the growth rate, the film thickness and perhaps even other more subtle parameters are all important in determining the growth mechanism and quality of the epitaxial layers. All the GaAs samples represented in this investigation have been grown by Lincoln Laboratory [13] and General Electric [14] using the typical parameters described here and in the references.

Numerous studies have demonstrated that as-grown LT-GaAs contains $\geqslant 1 \%$ excess arsenic, producing a large concentration of As antisite-related defects $\left(10^{18}-10^{20} \mathrm{~cm}^{-3}\right)$, and that it also has a $\approx 0.1 \%$ larger lattice constant than its bulk GaAs substrate $[15,16]$. The as-grown phase of LT-GaAs is known to have relatively low resistivity and mobility, so that even though it possesses a fast carrier response, it is not an outstanding photoconductor. When annealed (typically at $600{ }^{\circ} \mathrm{C}$ for $10-15 \mathrm{~min}$ ), the LT-GaAs displays a marked decrease in defects, and As precipitates with an average diameter of $60 \AA$ are observed by TEM to form within the single crystal host $|13,14|$. The crystallinity of the layer helps to maintain a high mobility, while the overlapping depletion regions of the metallic arsenic precipitates lead to a high resistivity. The material is then in a state that makes it ideal as a photoconductor, since besides its high resistivity and responsivity, it has a fast carrier response from the trapping/recombination sites [17] (perhaps with some contribution from the structural precipitate defects [18]) and a high dielectric breakdown as well [19]. What makes this material truly outstanding as a photoconductor, however, is that when it is optimally fabricated each of the above properties is superior to those of virtually all other materials. This is relevant not only for optical detection applications, but also for the characterization of electronics at the highest possible frequencies, since the $\approx 100 \mathrm{fs}$ carrier response of LT$\mathrm{GaAs}$ allows the generation of extremely short duration broadband electrical test signals [20].

Since the carrier lifetime of the LT-grown materials is so short, measurement techniques that have a temporal resolution high enough to characterize this response and the resulting high-bandwidth of LT-GaAs optoelectronic devices are necessary. While timeresolved photoluminescence would seem to be the most direct approach for determining carrier lifetimes, the high density of deep-level defects present in LT$\mathrm{GaAs}$, along with the high degree of non-radiative recombination of excited carriers in the material, makes this difficult [21]. Two alternative, but wellknown, high-resolution characterization techniques are used in the investigations described here: one which measures the optical response of LT-grown materials to excitation by a pulsed laser source, and another which determines the electrical response of a detector fabricated on an LT-grown material and illuminated by a short laser pulse.

The laser source primarily employed here is a solid state mode-locked titanium-doped sapphire (Ti:sapphire) oscillator with 65 fs duration pulses (FWHM), $350 \mathrm{~mW}$ average power, and $90 \mathrm{MHz}$ repetition rate. The pulse train from the laser is divided at a beam splitter, with part of the energy going into a pump beam and the rest to a probe beam. The pump pulses excite carriers in the LT-grown material under test through pair-breaking by above-bandgap energy photons. This carrier injection induces an absorption change in LT-GaAs through band filling, band gap renormalization and free carrier absorption [22]. This pump-induced change in absorption, $\Delta \alpha(t)$, and the 
corresponding change in reflection, evolve along with the carrier population due to a number of phenomena. Because most of these processes occur on a slow timescale, however, the relaxation decay of the reflection and the transmission (or $\Delta \alpha$ ) are influenced mainly by trapping/recombination at deep levels [23].

Figure 2 shows the normalized fractional transmissivity changes for two as-grown and two annealed LTGaAs samples grown at 190 and $200^{\circ} \mathrm{C}$. The carrier lifetime can be determined from the decay of the pulses to $1 / e$ of their peak amplitudes. These times are found to be extremely short, between 180 and $260 \mathrm{fs,} \mathrm{regard-}$ less of whether the material was annealed or not. This, along with other research [17], appears to provide evidence that the fast response of LT-GaAs may not be due to the precipitates, which are present only in the post-annealed material, but that deep levels arising from the excess arsenic or other point or structural defects are mainly responsible. Several studies also confirm that this ultrashort carrier lifetime cannot be sustained as the growth temperature is increased [23]. For instance, by the time growth temperatures are increased only to $250-300^{\circ} \mathrm{C}$, carrier lifetimes measured by this technique are typically $>50 \mathrm{ps}$.

The second measurement technique mentioned above, external electro-optic sampling (EOS), provides some information about the carrier lifetime of the LTgrown materials, but more importantly it is the only technique which can directly measure the sub-picosecond electrical response of a detector fabricated from LT-GaAs and related materials. It is not possible to resolve accurately an electrical signal having a rise time of less than even 10 ps with any conventional instrumentation available today. However, using short laser pulses and the very fast Pockels effect in an

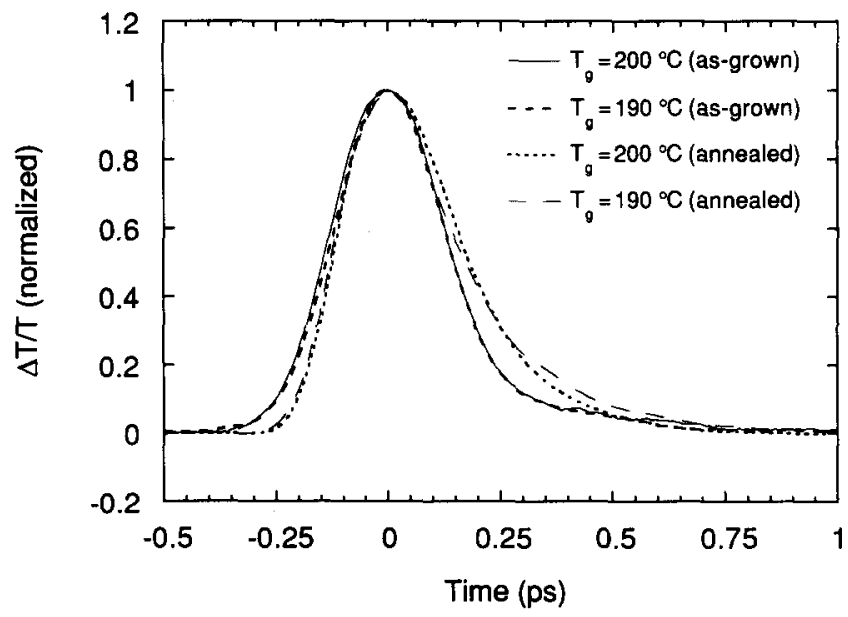

Fig. 2. Normalized fractional transmissivity of as-grown and annealed LT-GaAs layers grown at 190 and $200^{\circ} \mathrm{C}$. The data indicate photo-excited carrier lifetimes of less than $300 \mathrm{fs}$ in each case. electro-optic probe tip, it is possible to produce a measurement system with a bandwidth of $1 \mathrm{THz}$ [24].

The simplest detectors that are made have a gap within a planar transmission line or between two coplanar lines, fabricated on top of a fast photoconductor. The detector pattern most commonly used is a set of two parallel gold alloy metal lines, $20 \mu \mathrm{m}$ wide and separated by $20 \mu \mathrm{m}$, with a d.c. bias across the lines that is as high as $20-30 \mathrm{~V}$. The lasers pulses, of $\approx 20$ $\mu \mathrm{m}$ diameter, uniformly illuminate a spot between the lines to generate the electrical impulses. The electrical signals excited by the pump pulses are coupled from the detector to the transmission line and are readily measured using the EOS probe (Fig. 3). This photoconductive detector has very low capacitance, and thus the limitations on the shortness of the signal which can be generated arise from the duration of the laser pulse used or the lifetime of the photogenerated carriers. Radiation, skin-effect ohmic losses and modal dispersion lead to a rapid degradation of short impulses as they travel down the transmission line between the detector and the probe, however, often limiting the measurements to a resolution of around $500 \mathrm{fs}$. From Fig. 3 it can be seen that the determination of the carrier lifetime is limited compared with the pump-probe experiment. However, for the measurement of the impulse response of optoelectronic devices, the external EOS technique is invaluable.

\section{LT-GaAs photodetectors and photoswitches}

The previous section demonstrated that LT-GaAs, whether as-grown or annealed, has a tremendous

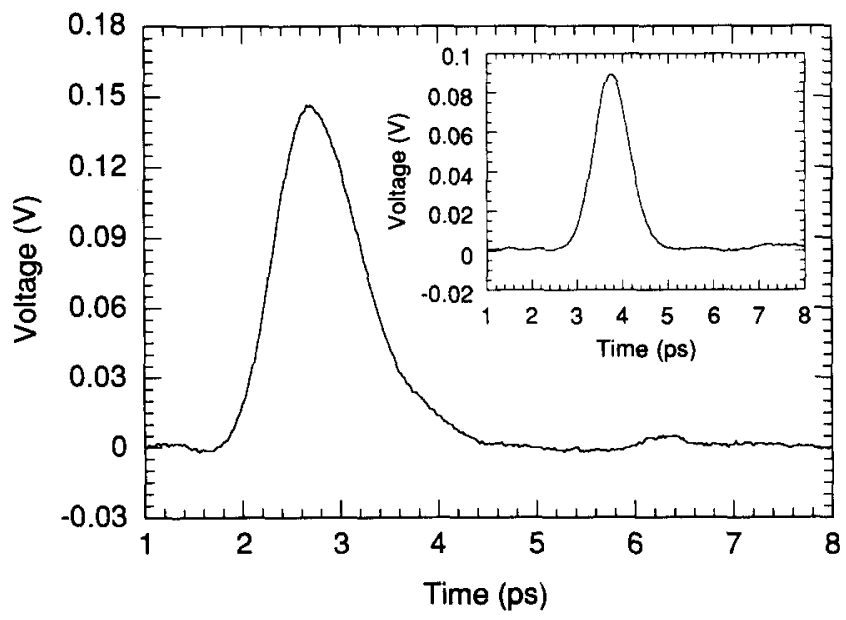

Fig. 3. Typical photoconductive response of simple detector gaps fabricated on annealed GaAs and (inset) as-grown LT-GaAs samples. The pulse widths are less than $1 \mathrm{ps}$ in duration, while the trailing edge returns to the baseline with virtually no tail in a comparable time. 
ability to respond to optical signals on a very short time-scale. Besides reproducing as closely as possible the temporal characteristics of an arbitrarily shaped optical signal, however, an ideal photodetector should, in general, be responsive to optical signals that may be very weak and also have a low conductivity or dark current in the absence of illumination. As mentioned in Section 2 , the resistivity of the as-grown LT-GaAs layers is relatively low, typically $10^{5}-10^{6} \Omega \mathrm{cm}$, as compared to the resistivity after annealing, which is $10^{7}-10^{8} \Omega \mathrm{cm}$. This leads to higher dark currents in detectors fabricated on unannealed LT-GaAs, as well as lower switching efficiencies and a diminished responsivity. For detector applications, even though a substrate of as-grown LT-GaAs provides a high-bandwidth device (Fig. 3, inset), its high conductivity hinders, and in some cases makes impossible, its use as a photoconductor.

While the most obvious approach is to exclusively utilize annealed LT-GaAs in detectors, several other experimental aspects independent of the annealing conditions have to be considered. The most important of these issues are the thickness of the LT-grown layer being employed in the detector and the wavelength of light that is being detected. For instance, the $1 / e$ penetration depth of the $800 \mathrm{~nm}$ light is around $1 \mu \mathrm{m}$, while that for $630 \mathrm{~nm}$ photons from a short-pulse dye laser is only about $0.3 \mu \mathrm{m}$. The ramifications for detection using a $1 \mu \mathrm{m}$ thick layer of LT-GaAs at these two wavelengths are shown in Fig. 4. While the $630 \mathrm{~nm}$ light is absorbed only in the LT-grown layer with the short carrier lifetime, the $800 \mathrm{~nm}$ light penetrates through the LT-GaAs and into the substrate, resulting in an electrical waveform that has the long lifetime characteristics of intrinsic GaAs, like the behaviour

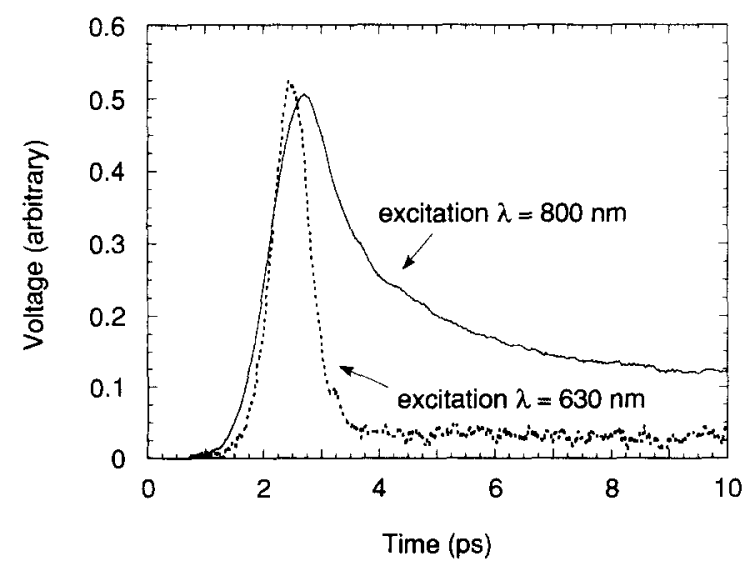

Fig. 4. Photoconductive response of a detector gap fabricated on a $1 \mu \mathrm{m}$ thick LT-GaAs layer for laser excitation wavelengths of 630 and $800 \mathrm{~nm}$. The longer wavelength penetrates farther into the detector structure, exciting long-lifetime carriers in the intrinsic $\mathrm{GaAs}$ substrate. seen in Fig. 1. This indicates that, for detection of longwavelength light, either a thicker LT-semiconductor layer must be grown, or a thin layer must be lifted off its substrate and grafted onto an insulator. This liftoff and grafting has been demonstrated by several groups $[25,26]$, and it gives LT-GaAs another advantage over other materials in optoelectronic applications, i.e. that a semiconductor with a fast carrier response can be bonded into circuits where a fast photoconductor could otherwise not be located. The high-frequency characteristics of several novel transmission line structures have been determined due to this flexibility of the LT-GaAs layers [27].

The benefits of a detector fabricated on a thicker LT-grown layer are substantiated in Fig. 5. Here the wavelength remains fixed at $800 \mathrm{~nm}$, while detectors fabricated on layers of different thickness, specifically 1 and $4.5 \mu \mathrm{m}$, are excited. Again, the penetration of longer wavelengths is found to degrade the response of a detector on a thin LT-GaAs layer. However, with a thick layer of LT-GaAs, the light penetration into the long lifetime substrate is negligible, and the response of the detector is very fast. The switching responsivity of the detectors is also found to be improved, by about a factor of three, when more of the light is absorbed in the LT-GaAs layer, i.e. when it is thicker. Therefore, a faster, more sensitive, optoelectronic device is realized when a thicker layer of LT-grown material is used for these simple, straight gap detectors.

The final characteristic of a detector which must be addressed is its ability to detect weak optical signals. Until the development of LT-GaAs, research on highspeed photodetectors concentrated on metal-semiconductor-metal (MSM) Schottky barrier photodiodes, with a device having $105 \mathrm{GHz}, 3 \mathrm{~dB}$ bandwidth and a

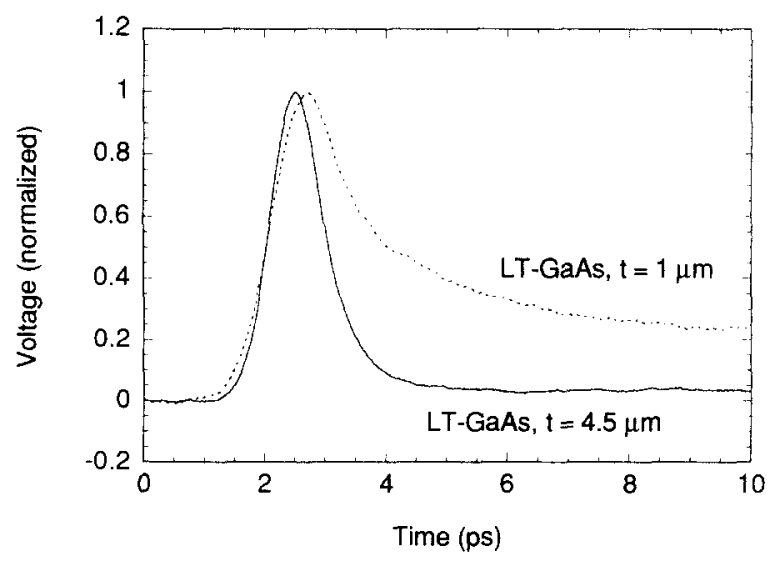

Fig. 5. Photoconductive response of simple detector gaps fabricated on $1 \mu \mathrm{m}$ and $4.5 \mu \mathrm{m}$ thick LT-GaAs layers for laser excitation wavelengths of $800 \mathrm{~nm}$. The light is mainly absorbed in the short-lifetime material only when the layer is substantially thicker than the penetration depth. 
$0.1 \mathrm{~A} \mathrm{~W}^{-1}$ responsivity being the best demonstrated [28]. With the small spacing of interdigitated fingers on this MSM detector, the short transit time of photoexcited carriers allows the metal electrodes to have an enhanced collection efficiency, increasing the conduction current. However, the slow recombination of the carriers in the undoped GaAs substrate and the low mobility of the holes limits the bandwidth of this detector.

When a finely spaced interdigitated patterndesigned so that the transit time is made equal to the carrier lifetime-is fabricated on the high-resistivity, annealed LT-GaAs, a photoconductive detector with a characteristic ultrahigh bandwidth and a responsivity normally associated with a photodiode can be produced. Such an MSM detector fabricated on LT-GaAs with $200 \mathrm{~nm}$ finger widths and spacings and an active area of $7 \mu \mathrm{m} \times 7 \mu \mathrm{m}$ is seen in Fig. 6. As with the simple detector gaps, it is defined between two coplanar metal lines. With an incident optical energy of $0.04 \mathrm{pJ}^{\text {pulse }} \mathrm{e}^{-1}$, this LT-GaAs detector has demonstrated a $3 \mathrm{~dB}$ bandwidth of $375 \mathrm{GHz}$ with a responsivity of $0.1 \mathrm{~A} \mathrm{~W}^{-1}$, making it the fastest high-sensitivity detector reported [29]. The dark current at a $1 \mathrm{~V}$ bias is $100 \mathrm{pA}$, and for a higher optical fluence of $22 \mathrm{pJ}_{\text {pulse }}{ }^{-1}$ the on-state resistance of the detector is as low as $30 \Omega$

This ability to drive the detector to such a low resistance state also allows the interdigitated gaps to be employed as sampling gates. In one investigation [23], two MSM structures were integrated on one wafer of LT-GaAs (Fig. 7, inset), where one behaved as the

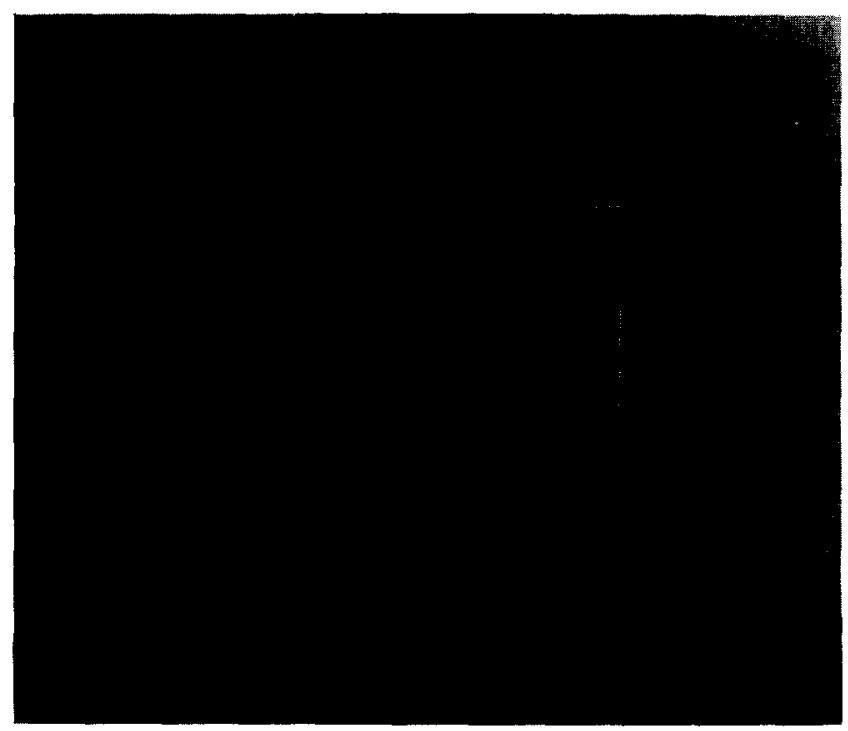

Fig. 6. Interdigitated MSM detector gap on annealed LT-GaAs with finger widths and spacings of $200 \mathrm{~nm}$ and area of $50 \mu \mathrm{m}^{2}$ (magnified $1700 \times$ ). Extremely high sensitivities with high bandwidths are combined in this structure. detector (in the shunt position), and one served as a sampling gate. The standard pump-probe configuration is again utilized, with the delay between the excitation and sampling pulses plotted against the signal amplitude. The MSM detector, which can measure even very weak optical transients, acts as a correlator if both the detected signal and the sampling gate signal are the same (Fig. 7), and as a time domain optical waveform analyzer if the transient illuminating the detector has an arbitrary pulse shape.

\section{Low-temperature-grown $\operatorname{In}_{x} \mathrm{Ga}_{1-x} \mathrm{As}$}

While all of the detectors reported in this review are excited using laser pulses at $\lambda=800$ or $630 \mathrm{~nm}$, the main commercial applications for high-speed detectors will be found in the operating regimes of fibre optic communications systems, around 1.3 and $1.5 \mu \mathrm{m}$. While LT-GaAs has actually been found to absorb 1.3 $\mu \mathrm{m}$ light [30], it is also desirable to investigate whether other LT-grown materials could be used in high-bandwidth detectors for communications. To reach the bandgap associated with $1.5 \mu \mathrm{m}$ light, a material such as $\mathrm{In}_{0.53} \mathrm{Ga}_{0.47} \mathrm{As}$, which is sensitive down to $1.7 \mu \mathrm{m}$, is needed. Unfortunately, even though $\mathrm{In}_{0.53} \mathrm{Ga}_{0.47} \mathrm{As}$ grown on InP has been demonstrated to have a carrier lifetime of $2.5 \mathrm{ps}$ when grown at $180^{\circ} \mathrm{C}$ [23], it has a very low resistivity and thus is not a candidate for use in high-speed infrared detectors. It has been recently discovered, however, that LT- $\operatorname{In}_{x} \mathrm{Ga}_{1-x}$ As grown lattice-mismatched on GaAs, with InAs mole fractions as high as $35 \%$, does have a moderately high resistivity to go along with a picosecond carrier lifetime [23]. As

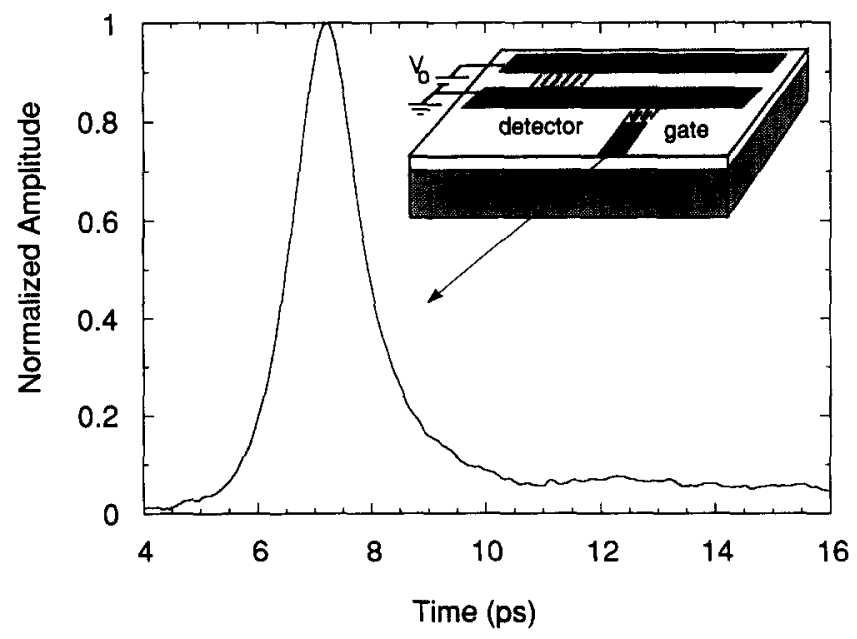

Fig. 7. Electrical response of two interdigitated photoconductive gaps fabricated on annealed LT-GaAs for use as integrated detector and sampling gate (inset). The FWHM response is 1.4 ps. 


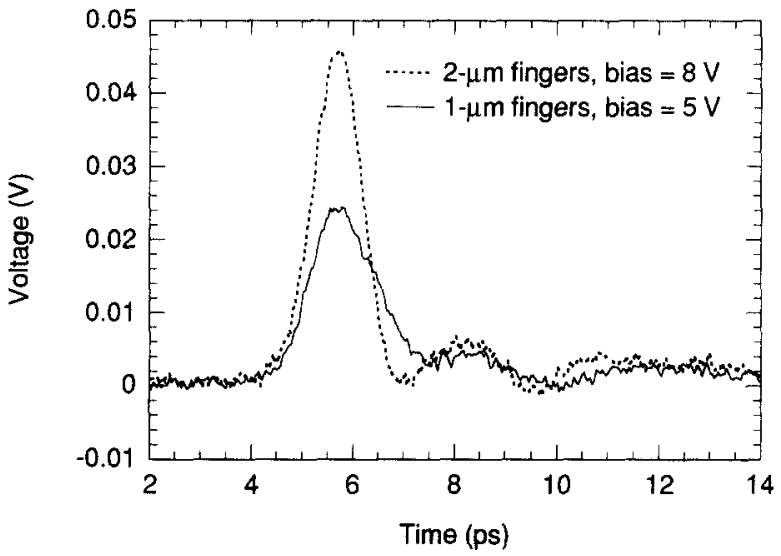

Fig. 8. Photoconductive response of interdigitated detectors fabricated on LT- $\mathrm{In}_{0.35} \mathrm{Ga}_{0.65} \mathrm{As}$ on GaAs, with $1 \mu \mathrm{m}$ and $2 \mu \mathrm{m}$ features, for a laser excitation wavelength of $800 \mathrm{~nm}$.

in the LT-GaAs system, the carrier lifetime for the LT$\operatorname{In}_{x} \mathrm{Ga}_{1-x}$ As is shortest $(\approx 1 \mathrm{ps})$ at the lowest growth temperatures used $\left(<200^{\circ} \mathrm{C}\right)$, and it increases (to $>10$ ps) at higher growth temperatures $\left(250-400^{\circ} \mathrm{C}\right)$. Annealing of the material also leads to the formation of precipitates, although compared with LT-GaAs, the behaviour of $\mathrm{LT}-\mathrm{In}_{x} \mathrm{Ga}_{1-x}$ As is much more difficult to predict, since the anneal relaxes the strain and some of the structural defects which arise from the lattice mismatch.

Thus far, $1 \mu \mathrm{m}$ thick layers of $\mathrm{LT}-\operatorname{In}_{x} \mathrm{Ga}_{1-x} \mathrm{As}$ having both $x=0.25$ and $x=0.35$ have been grown and tested for carrier response and utility as a detector substrate [31]. While the $25 \%$ InAs mole fraction material appears to have a resistivity advantage, its bandgap is too large to detect radiation with $\lambda>1.1 \mu \mathrm{m}$ directly. The LT- $\mathrm{In}_{0.35} \mathrm{Ga}_{0.65} \mathrm{As}$ does have a cutoff at $1.3 \mu \mathrm{m}$, however, and thus applications are now concentrating on the use of LT- $\operatorname{In}_{x} \mathrm{Ga}_{1-x}$ As with this composition. In one preliminary experiment using LT- $\mathrm{In}_{0.35} \mathrm{Ga}_{0.65} \mathrm{As}$, an interdigitated pattern having finger widths and separations of $1 \mu \mathrm{m}$ and an area of $13 \mu \times 20 \mu \mathrm{m}$ was fabricated [31]. The layer used was grown at a temperature of $\approx 125^{\circ} \mathrm{C}$ and was thus of poor crystal quality (in fact, it was believed to be amorphous). Nevertheless, a picosecond duration response was observed from this detector (Fig. 8), albeit with a depressed responsivity of $3 \times 10^{-4} \mathrm{~A} \mathrm{~W}^{-1}$. The fabrication of a larger pattern, having $2 \mu \mathrm{m}$ finger widths and separations, allowed the use of a larger voltage bias $\left(8 \mathrm{~V}\right.$, or $\left.40 \mathrm{kV} \mathrm{cm}^{-1}\right)$, and thus an even larger detector response was obtained. With optimization of the material properties, and other improvements such as the use of antireflection coatings, it is believed that LT- $\operatorname{In}_{x} \mathrm{Ga}_{1-x}$ As can form the foundation of a family of long-wavelength, high-sensitivity, high-bandwidth detectors.

\section{Conclusions}

The behaviour of the carrier lifetime of LT-grown layers, as well as the influence of wavelength, layer thickness, and annealing conditions on LT-GaAs detectors, have been reviewed. The ultrafast carrier response of LT-GaAs and $\operatorname{In}_{x} \mathrm{Ga}_{1-x}$ As has been demonstrated to be of tremendous utility for highbandwidth optoelectronic applications. Advanced detection and gating devices based on MSM interdigitated structures fabricated on LT-GaAs have also been shown to have superior performance compared to similar semiconductor devices. The potential for using LT-In ${ }_{x} \mathrm{Ga}_{1-x} \mathrm{As} / \mathrm{GaAs}$ in high-speed, long-wavelength detector applications for optical communications systems has also been established.

\section{Acknowledgments}

Many past and present staff members and graduate students at the University of Michigan contributed to the work reported here, including S. L. Williamson, S. Gupta, J. A. Nees, T. Brock, G. A. Mourou, H.-H. Wang, H.-J. Cheng, Y. Chen and J. Kim. We thank F. W. Smith of Lincoln Laboratory and J. M. Ballingall of General Electric for their expert preparation of the LTgrown semiconductor films used. This research has been supported by AFOSR contract no. AFOSR-900214 (University Research Initiative) and by the National Science Foundation through the Center for Ultrafast Optical Science under STC PHY 8920108.

\section{References}

1 D. H. Auston, Appl. Phys. Lett., 26 (1975) 101.

2 C. H. Lee, Appl. Phys. Lett., 30 (1977) 84.

3 T. Y. Hsiang, J. F. Whitaker, R. Sobolewski, D. R. Dykaar and G. A. Mourou, Appl. Phys. Lett., 51 (1987) 1551.

4 M. K. Jackson, M. Y. Frankel, J. F. Whitaker, G. A. Mourou, D. Hulin. A. Antonetti, M. Van Hove, W. De Raedt, P. Crozat and H. Hafdallah, Appl. Phys. Lett., 61 (1992) 1187.

5 D. Krökel, D. Grischkowsky and M. B. Ketchen, Appl. Phys. Lett., 54 (1989) 1046.

6 M. Y. Frankel, S. Gupta, J. A. Valdmanis and G. A. Mourou, Electron. Lett., 25 (1989) 1363.

7 W. C. Nunnally and R. B. Hammond, in C. H. Lee (ed.), Picosecond Electronics, Academic Press, Orlando, 1984, p. 373.

8 M. C. Nuss, D. W. Kisker, P. R. Smith and T. E. Harvey, Appl. Phys. Lett., 54 (1989) 57.

9 F. E. Doany, D. Grischkowsky and C. -C. Chi, Appl: Phys. Lett., $50(1987) 460$.

10 B. Lambsdorff, J. Kuhl, J. Rosenzweig, A. Axmann and J. Schneider, Appl. Phys. Lett., 58 (1991) 1881.

11 A. G. Foyt, F. J. Leonberger and R. C. Williamson, Appl. Phys. Lett., 40 (1982) 448.

12 F. W. Smith, S. Gupta, H. Q. Le, V. Diadiuk, M. A. Hollis, A. R. Calawa, M. Frankel, D. R. Dykaar, G. A. Mourou and T. Y. Hsiang, Appl. Phys. Lett., 54 (1989) 890. 
13 S. Gupta, M. Y. Frankel, J. A. Valdmanis, J. F. Whitaker, G. A. Mourou, F. W. Smith and A. R. Calawa, Appl. Phys. Lett., 59 (1991) 3276 .

14 H. H. Wang, J. F. Whitaker, A. Chin, J. Mazurowski and J. M. Ballingall, J. Electron. Mater., in press.

15 M. Kaminska, Z. Liliental-Weber, E. R. Weber, T. George, J. B. Kortright, F. W. Smith, B.-Y. Tsaur and A. R. Calawa, Appl. Phys. Lett., 54 (1989) 1881.

16 A. C. Warren, J. M. Woodall, J. L. Freeouf, D. Grischkowsky, D. T. McInturff, M. R. Melloch and N. Otsuka, Appl. Phys. Lett., $57(1990) 1331$.

17 Z. Liliental-Weber, H. J. Cheng, S. Gupta, J. F. Whitaker, K. Nichols and F. W. Smith, J. Electron. Mater, in press.

18 A. C. Warren, N. Katzenellenbogen, D. Grischkowsky, J. M. Woodall, M. R. Melloch and N. Otsuka, Appl. Phys. Lett., 58 (1991) 1512

19 M. Y. Frankel, J. F. Whitaker, G. A. Mourou, F. W. Smith and A. R. Calawa, IEEE Trans. Electron. Dev., 37 (1990) 2493.

$20 \mathrm{M}$. Y. Frankel, J. F. Whitaker and G. A. Mourou, IEEE J. Quantum Electron., 28(1992) 2313.

21 H. M. van Driel, X.- Q. Zhou, W. W. Rühle, J. Kuhl and K. Ploog, Appl. Phys. Lett., 60 (1992) 2246.
22 B. R. Bennett, R. A. Soref, and J. A. del Alamo, IEEE J Quantum Electron., 26 (1990) 113.

23 S. Gupta, J. F. Whitaker and G. Mourou, IEEE J. Quantum Electron., 28 (1992) 2464.

24 J. A. Valdmanis and G. Mourou, IEEE J. Quantum Electron., 22 (1986) 69.

25 E. Yablonovitch, D. Hwang, T. Gmitter, L. Florez and J. Harbison, Appl. Phys. Lett., 56 (1990) 2419.

$26 \mathrm{H}$. J. Cheng and J. F. Whitaker, in J. Shah (ed.), Ultrafast Electronics and Optoelectronics, OSA, Washington, in press.

27 H. J. Cheng and J. F. Whitaker, in IEEE MTT-S International Microwave Symposium Digest, IEEE, New York, Vol. 3, 1993, p. 1355.

28 B. J. van Zeghbroeck, W. Patrick, J.-M. Halbout and P. Vettiger, IEEE Electron. Dev. Lett., 9 (1988) 527.

29 Y. Chen, S. Williamson, T. Brock, F. W. Smith and A. R. Calawa, Appl. Phys. Lett., 59 (1991) 1984.

30 A. C. Warren, J. H. Burroughes, J. M. Woodall, D. T. McInturff, R. T. Hodgson and M. R. Melloch, IEEE Electron. Dev. Lett., 12 (1991) 527.

31 L. F. Lester, K. C. Hwang, P. Ho, J. Mazurowski, J. M. Ballingall, J. Sutliff, S. Gupta, J. F. Whitaker and S. L. Williamson, IEEE Photon. Tech. Lett., 5 (1993) 511. 